\title{
Silencing of phosphoinositide dependent protein kinase orthologs reduces hypersensitive cell death in Nicotiana benthamiana
}

\author{
Akinori Kiba ${ }^{1, *}$, Kotoko Fukui ${ }^{1}$, Maki Mitani ${ }^{1}$, Ivan Galis ${ }^{2}$, Yuko Hojo ${ }^{2}$, \\ Tomonori Shinya ${ }^{2}$, Kouhei Ohnishi ${ }^{3}$, Yasufumi Hikichi ${ }^{1}$ \\ ${ }^{1}$ Laboratory of Plant Pathology and Biotechnology, Faculty of Agriculture, Kochi University, Nankoku, Kochi 783-8502, Japan; \\ ${ }^{2}$ Institute of Plant Science and Resources, Okayama University, Okayama 710-0046, Japan; ${ }^{3}$ Research Institute of Molecular \\ Genetics, Kochi University, Nankoku, Kochi 783-8502, Japan \\ *E-mail: akiba@kochi-u.ac.jp Tel \& Fax:+81-88-864-5196
}

Received April 1, 2020; accepted May 11, 2020 (Edited by M. Sekine)

\begin{abstract}
Phosphatidic acid plays an important role in plant immune responses against phytopathogenic bacteria in Nicotiana benthamiana. Here we focused on phosphoinositide dependent protein kinases (PDKs) as a candidate required for phosphatidic acid signaling. Based on Arabidopsis PDK sequences, we identified four putative PDK orthologs in $N$. benthamiana genome. To address the role of PDKs in plant defense responses, we created all four NbPDKs-silenced plants by virus-induced gene silencing. the $N b P D K s$-silenced plants showed a moderately reduced growth phenotype. Induction of hypersensitive cell death was compromised in the NbPDKs-silenced plants challenged with Ralstonia solanacearum. The hypersensitive cell death induced by bacterial effectors was also reduced in the NbPDKs-silenced plants. the NbPDKssilenced plants showed decreased production of salicylic acid, jasmonic acid and jasmonoyl-L-isoleucine, as well as hydrogen peroxide after inoculation with $R$. solanacearum. These results suggest that NbPDKs might have an important role in the regulation of the hypersensitive cell death via plant hormone signaling and oxidative burst.
\end{abstract}

Key words: effector-triggered immunity, hypersensitive cell death, Nicotiana benthamiana, phosphoinositide dependent protein kinase, Ralstonia solanacearum, virus-induced gene silencing.

It has been shown that phospholipid-based signaling cascades are important for plant immune responses (Canonne et al. 2011; Munnik 2001; Testerink and Munnik 2005). Recently, we identified the SEC14 gene encoding a phospholipid transfer protein from $N$. benthamiana (NbSEC14). Suppression of phospholipase $\mathrm{C}$ and phospholipase D activity, and production of diacylglycerol and phosphatidic acid (PA) were observed in NbSEC14-silenced plants, resulting in compromised disease resistance against phytopathogenic bacteria (Kiba et al. 2012, 2014, 2016). Silencing of PA phosphatase caused over accumulation of PA, resulting in enhanced disease resistance against Ralstonia solanacearum (Nakano et al. 2013). Induction of hypersensitive cell death (HCD) was also accelerated in PA phosphatasesilenced plants (Nakano et al. 2015). These results suggest an important role of PA in the induction of immune responses and HCD in N. benthamiana. To this end, PA functions as a signal molecule in responses to both biotic and abiotic stresses (Liu et al. 2013).
Previously, PA production was activated by osmotic stresses, such as salinity, drought, hyperosmotic stress, and treatment with the drought-related stress hormone abscisic acid (Munnik 2001). PA is also formed after wounding of leaves in various plants, including soybean, sunflower, pepper, broad bean, and castor bean (Munnik 2001; Testerink and Munnik 2005). The chitin elicitor induces the PA biosynthesis, leading to phytoalexin accumulation in rice cells (Yamaguchi et al. 2005), and PA is also produced after Cf-4 mediated pathogen recognition (de Jong et al. 2004). Taken together, PA has an important role in protection of plants against abiotic and biotic stresses. Recently, PAbinding proteins have been characterized as the targets of PA, being involved in the regulation of plant growth, development, and stress responses (Liu et al. 2013; McLoughlin and Testerink 2013). While many proteins with PA-binding properties have been identified mainly in Arabidopsis thaliana, it is still poorly understood how PA-binding proteins function in $N$. benthamiana and $R$.

Abbreviations: DAB, 3,3'-diaminobenzidine; ETI, Effector-triggered immunity; HCD, hypersensitive cell death; PA, phosphatidic acid; PDK, phosphoinositide dependent protein kinase; Rs, Ralstonia solanacearum; VIGS, virus-induced gene silencing.

This article can be found at http://www.jspcmb.jp/

Published online July 30, 2020 
solanacearum interaction. In this study, we focused on phosphoinositide dependent kinases (PDKs) from $N$. benthamiana as candidates for PA-mediated signaling components required for immune responses against $R$. solanacearum, namely HCD induction.

Nicotiana benthamiana was grown in a cultivation room as before (Maimbo et al. 2007). Ralstonia solanacearum strain 8107 (Rs8107), which is an incompatible pathogen inducing HCD on $N$. benthamiana (Kiba et al. 2003) and Agrobacterium tumefaciens were cultured as described previously (Maimbo et al. 2010). The bacterial suspensions were infiltrated by syringes using standard procedures (Maimbo et al. 2010). Total RNA was isolated using a NucleoSpin RNA Plant kit (Macherey-Nagel, Düren, Germany). A 1- $\mu$ g sample of total RNA was used as the template for reverse transcription with a ReverTra Ace $^{\circledR}$ qPCR RT Kit (Toyobo Co., Ltd. Tokyo, Japan). The sequence analysis was performed with the reagents for the Big Dye Terminator Cycle Sequencing Kit (Applied Biosystems, Foster, CA, USA) and an Applied Biosystems 3100 Avant Automated Sequencer (Applied Biosystems, Warrington, UK). cDNA fragments for the conserved region of NbPDK2-1 to NbPDK2-4 sequences $(N b P D K s)$ were amplified with specific pairs of primers (Supplementary Figure S1, Table S1), and then cloned into the pPVX201 (Kiba et al. 2012). The binary plasmids were transformed into A. tumefaciens strain GV3101, and the transformant was inoculated into $N$. benthamiana leaves as described previously (Maimbo et al. 2007). qRT-PCR was performed using the method described in Maimbo et al. (2007). Briefly, the qRT-PCR was carried out in $20 \mu \mathrm{l}$ of reaction mixture, containing $1 \mu \mathrm{l}$ of cDNA template, $10 \mathrm{pM}$ of the respective primers (Supplementary Table S1) and THUNDERBIRD qPCR MIX (Toyobo Co.), on an Applied Biosystems 7300 realtime PCR instrument. All values were normalized to the expression values of the actin gene, which was used as an internal standard in each cDNA stock. Cell death was measured by ion conductivity method (Ito et al. 2014a, b) using a Twin Cord B-173 conductivity meter (HORIBA, Kyoto, Japan). Phytohormone contents were determined by a triple quadrupole Liquid Chromatography/Mass Spectrometer LC-MS/MS 6410 (Agilent Technologies, USA) equipped with a Zorbax SB-C18 column [2.1 $\mathrm{mm}$ i.d. $\times 50 \mathrm{~mm},(1.8 \mu \mathrm{m})$, Agilent Technologies]. Hormone amounts were calculated from the ratio of the endogenous hormone peak and the known amounts of stable isotope labeled internal standards, and related to the actual fresh mass of the samples used for extraction (Kiba et al. 2014). Visualization of $\mathrm{H}_{2} \mathrm{O}_{2}$ in situ was carried out by $3,3^{\prime}$-diaminobenzidine (DAB) staining as described by Nakano et al. (2013).

Based on PDK1 sequences from Arabidopsis thaliana, we searched for PDK orthologs in N. benthamiana using the sol genomics network (https://solgenomics.net/). We identified 4 PDK orthologs in the N. benthamiana genome. Database analysis of PDK orthologs showed high similarity to PDK2 from Nicotiana and other plants, and we therefore designated them as NbPDK2-1, NbPDK2-2, NbPDK2-3 and NbPDK2-4 (Supplementary Figure S1A, B). Deduced amino acid sequences of the full-length cDNAs of the NbPDK2-1, NbPD2-2 and NbPDK2-3 contained STKc-PDK1 and PH domains. In contrast, NbPDK2-4 lacked half of STKc-PDK1 and PH domains (Supplementary Figure S1A, C). In Arabidopsis, PDK1 was activated by binding of $\mathrm{PA}$ to $\mathrm{PH}$ domain, and STKc-PDK1 was required for the enzymatic activity (Anthony et al. 2004). In the present study, NbPDK2-1, 2 and 3 contained conserved both STKc-PDK1 and PH domain, suggesting that NbPDK2-1, 2 and/or 3 act as signaling component downstream of PA (Supplementary Figure S1).

Then, we created constructs for obtaining all four $P D K s$ (NbPDKs)-silenced plants (Supplementary Figure S2). Suppression of all NbPDKs after initiation of VIGS was estimated by qRT-PCR (Supplementary Figure S3A). The NbPDKs-silenced plants showed moderately dwarf phenotype (Supplementary Figure S3B, C), consistent with previous reports on phospholipid signaling having a role in plant growth (Xue et al. 2007). Therefore, our NbPDKs might also act as downstream phospholipid signaling components during plant growth. To address the role of NbPDKs in plant defense responses, we inoculated Rs8107 to clarify the silencing effect of NbPDKs on HCD. The induced cell death appeared in the control plants $18-24 \mathrm{~h}$ after inoculation, whereas suppression of cell death was observed in NbPDKssilenced plants (Figure 1A). The induced level of HCD marker hin1 gene was also suppressed in NbPDKssilenced plants (Figure 1B). These results suggested that NbPDK2s act as positive regulator for HCD in $N$. benthamiana (Figure 1). In tomato, PDK1 phosphorylates cell death inhibitor Adi3, and gene silencing of Adi3 caused MAPK dependent dell death. Chemical inhibitor of PDK1, OSU-03012, also reduced viability of tomato protoplasts (Devarenne et al. 2006), indicating a negative regulation of HCD by PDK1 in tomato cells. Therefore, the role of PDKs in HCD regulation may be different between $N$. benthamiana plants and tomato plants. In addition, silencing the NbPDKs also had inhibitory effect on the induction of HCD by the Agrobacterium-mediated transient expression of R. solanacearum effectors, AvrA and PopP1 (Poueymiro et al. 2009, Figure 1C). Plants have evolved the innate immune system to recognize pathogens effector proteins by their cognate resistance proteins, resulting in the initiation of effector-triggered immunity (ETI) (Gassmann and Bhattacharjee 2012). ETI is characterized as specific, sustainable and robust immune 
A

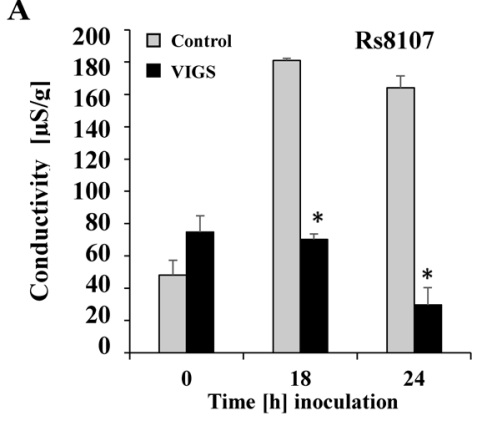

C

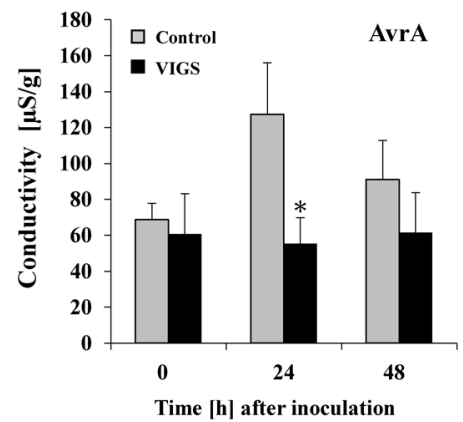

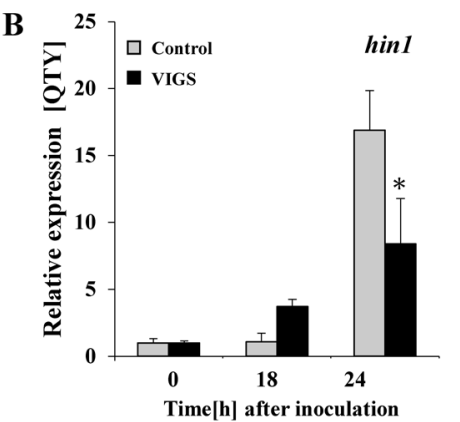

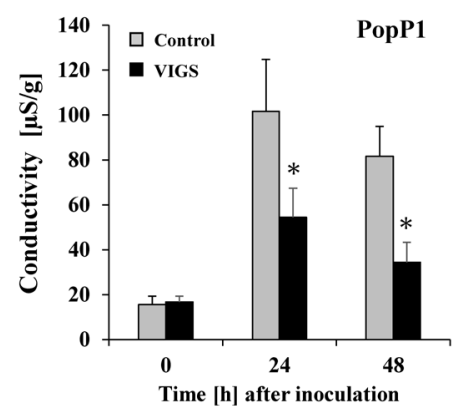

Figure 1. Effect of NbPDKs-silencing on HCD induction. Control and NbPDKs-silenced N. benthamiana plants were infiltrated with $R$. solanacearum 8107 (Rs8107), Agrobacterium harboring 35S-AvrA (AvrA) and 35S-PopP1 (PopP1). (A) Cell death induced by R. solanacearum was determined by measurement of ion conductivity. Values represent mean with SD $(n=5)$. (B) Total RNA was isolated from $R$. solanacearum inoculated plants, and relative expression of Nbhin1 transcripts relative to the absolute non-treated control, and normalized with actin (hin 1). Values represent the means and SD from triplicate experiments. (C) Cell death induction by AvrA and PopP1 were determined by measurement of ion conductivity. Values represent mean with SD $(n=5)$.

A

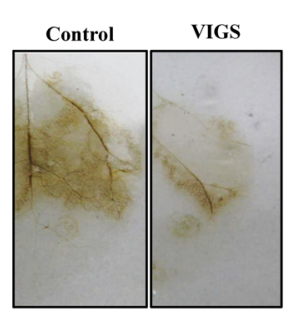

B

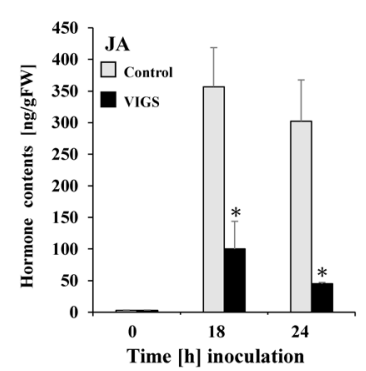

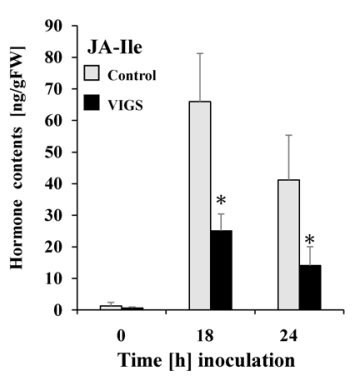

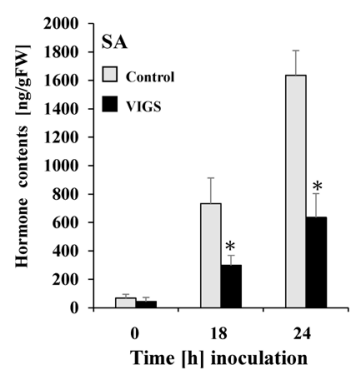

Figure 2. Effect of NbPDKs-silencing on oxidative burst and plant hormone synthesis. Control and NbPDKs-silenced N. benthamiana plants were infiltrated with $R$. solanacearum 8107. (A) Hydrogen peroxide was visualized $18 \mathrm{~h}$ after inoculation with $R$. solanacearum by DAB staining as described in Materials and Methods. (B) Total jasmonic acid (JA), jasmonoyl-L-isoleucine (JA-Ile), and salicylic acid (SA) content were determined at designated time points. Values represent the means and SE from replicated experiments $(n=5)$. Asterisks denote values significantly different from those of control plants $(* ; p<0.05, t$-test).

response with HCD (Jones and Dangl 2006). Therefore, our results demonstrate that NbPDKs contributes to induction of HCD in responses to Rs8107, as well as effectors-induced HCD, suggesting the involvement of NbPDKs in ETI induction.

During the HCD induction by Rs8107 inoculation, hydrogen peroxide production was detected in control plants. In contrast, reduced hydrogen peroxide levels were observed in NbPDKs-silenced plants (Figure 2A). Reactive oxygen species reportedly play a role in HCD induction (Torres 2010; Yoshioka et al. 2003; Zhang et al. 2009). It has been reported that activated PDK1 stimulates OXI1/AGC2-1 required for oxidative signaling in A. thaliana (Anthony et al. 2006). The PA-PDK1 signaling cascade also induces a respiratory burst in plants (Rentel et al. 2004; Yu et al. 2010). Our present data confirm the involvement of NbPDKs in triggering reactive oxygen production (Figure $2 \mathrm{~A}$ ), and therefore, NbPDKs might be playing an important role in HCD regulation via oxidative signals.

As shown in Figure 2B, jasmonic acid (JA), JA-Lisoleucine, as well as salicylic acid (SA) accumulated in control plants by 18 and $24 \mathrm{~h}$ after inoculation with Rs8107, whereas reduced levels of all plant hormones 
were observed in NbPDKs-silenced plants. Plant hormonal signals are critical for HCD induction. In tomato plants, JA signaling pathway is reportedly required for HCD-inducible $\beta$-cryptogein signaling (Starý et al. 2019). The increased resistance against powdery mildew was correlated with cell death, $\mathrm{H}_{2} \mathrm{O}_{2}$ accumulation, and upregulated expression of SAdependent defense genes in $N$. benthamiana (Guo et al. 2019). Now, our present data also show involvement of NbPDKs in JA and SA signaling at the level of biosynthesis of these hormone (Figure 2B). Therefore, NbPDKs might be having an important role in HCD induction, via regulation of JA and SA biosynthetic pathways.

In summary, we demonstrate that NbPDKs contribute to induction of HCD in plants. From our findings, while undergoing ETI induction, NbPDKs may be activating oxidative burst, JA and SA-mediated signaling in plants, during HCD induction. Further studies will be required to clarify the role of individual PDKs (NbPDK2-1 to 2-4) and complex mechanisms by which the NbPDK proteins are engaged in HCD induction.

\section{Acknowledgements}

The authors thank S. Genin for the provision of P35S-PopP1 and P35S-AvrA, and D. Baulcombe for the pPVX201 vector. This work was supported by Cabinet Office grant in aid, the Advanced Next-Generation Greenhouse Horticulture by IoP (Internet of Plants), Japan. This research was also supported by the Ministry of Education, Culture, Sports, Science and Technology (MEXT) as part of Joint Research Program implemented at the Institute of Plant Science and Resources, Okayama University in Japan. LCMS/MS instrumentation was supported by the Japan Advanced Plant Science Network. AK is also grateful for financial support from Grants in Aid for Scientific Research to AK (24580066) from the Ministry of Education, Science, Sports, and Culture, Japan, The Asahi Glass Foundation, The Agricultural Chemical Research Foundation and Sapporo Bioscience Foundation.

\section{Author contributions}

A.K., K.O. and Y.H. designed the research; A.K., K.F., M.M., I.G. and Y.H. performed the research; A.K., I.G. and T.S. analyzed the data and wrote the article.

\section{References}

Anthony RG, Henriques R, Helfer A, Meszaros T, Rios G, Testerink C, Munnik T, Deák M, Koncz C, Bögre L (2004) A protein kinase target of a PDK1signalling pathway is involved in root hair growth in Arabidopsis. EMBO J 23: 572-581

Anthony RG, Khan S, Costa J, Pais MS, Bögre L (2006) The Arabidopsis protein kinase PTI1-2 is activated by convergent phosphatidic acid and oxidative stress signaling pathways downstream of PDK1 and OXI1. J Biol Chem 281: 37536-37546

Canonne J, Froidure-Nicolas S, Rivas S (2011) Phospholipases in action during plant defense signaling. Plant Signal Behav 6:
$13-18$

de Jong CF, Laxalt AM, Bargmann BO, de Wit PJ, Joosten $\mathrm{MH}$, Munnik T (2004) Phosphatidic acid accumulation is an early response in the Cf-4/Avr4 interaction. Plant J 39: 1-12

Devarenne TP, Ekengren SK, Pedley KF, Martin GB (2006) Adi3 is a Pdk1-interacting AGC kinase that negatively regulates plant cell death. EMBO J 25: 255-265

Gassmann W, Bhattacharjee S (2012) Effector-triggered immunity signaling: From gene-for-gene pathways to protein-protein interaction networks. Mol Plant Microbe Interact 25: 862-868

Guo WL, Chen BH, Guo YY, Yang HL, Mu JY, Wang YL, Li XZ, Zhou JG (2019) Improved powdery mildew resistance of transgenic Nicotiana benthamiana overexpressing the Cucurbita moschata CmSGT1 Gene. Front Plant Sci 10: 955

Ito M, Takahashi H, Sawasaki T, Ohnishi K, Hikichi Y, Kiba A (2014a) Novel type of adenylyl cyclase participates in tabtoxinine- $\beta$-lactam-induced cell death and occurrence of wildfire disease in Nicotiana benthamiana. Plant Signal Behav 9: e27420

Ito M, Yamamoto Y, Kim CS, Ohnishi K, Hikichi Y, Kiba A (2014b) Heat shock protein 70 is required for tabtoxinine- $\beta$-lactaminduced cell death in Nicotiana benthamiana. J Plant Physiol 171: 173-178

Jones JD, Dangl JL (2006) The plant immune system. Nature 444: 323-329

Kiba A, Galis I, Hojo Y, Ohnishi K, Yoshioka H, Hikichi Y (2014) SEC14 phospholipid transfer protein is involved in lipid signaling-mediated plant immune responses in Nicotiana benthamiana. PLoS One 9: e98150

Kiba A, Imanaka Y, Nakano M, Galis I, Hojo Y, Shinya T, Ohnishi K, Hikichi Y (2016) Silencing of Nicotiana benthamiana SEC14 phospholipid transfer protein reduced jasmonic acid dependent defense against Pseudomonas syringae. Plant Biotechnol 33: 111-115

Kiba A, Nakano M, Vincent-Pope P, Takahashi H, Sawasaki T, Endo Y, Ohnishi K, Yoshioka H, Hikichi Y (2012) A novel Sec14 phospholipid transfer protein from Nicotiana benthamiana is up-regulated in response to Ralstonia solanacearum infection, pathogen associated molecular patterns and effector molecules and involved in plant immunity. J Plant Physiol 169: 1017-1022

Kiba A, Tomiyama H, Takahashi H, Hamada H, Ohnishi K, Okuno T, Hikichi Y (2003) Induction of resistance and expression of defense-related genes in tobacco leaves infiltrated with Ralstonia solanacearum. Plant Cell Physiol 44: 287-295

Liu Y, Su Y, Wang X (2013) Phosphatidic acid-mediated signaling. Adv Exp Med Biol 991: 159-176

Maimbo M, Ohnishi K, Hikichi Y, Yoshioka H, Kiba A (2007) Induction of a small heat shock protein and its functional roles in Nicotiana Plants in the defense response against Ralstonia solanacearum. Plant Physiol 145: 1588-1599

Maimbo M, Ohnishi K, Hikichi Y, Yoshioka H, Kiba A (2010) $S$ glycoprotein-like protein regulates defense responses in Nicotiana plants against Ralstonia solanacearum. Plant Physiol 152: 2023-2035

McLoughlin F, Testerink C (2013) Phosphatidic acid, a versatile water-stress signal in roots. Front Plant Sci 4: 525

Munnik T (2001) Phosphatidic acid: An emerging plant lipid second messenger. Trends Plant Sci 6: 227-233

Nakano M, Nishihara M, Yoshioka H, Takahashi H, Sawasaki T, Ohnishi K, Hikichi Y, Kiba A (2013) Suppression of DS1 phosphatidic acid phosphatase confirms resistance to Ralstonia solanacearum in Nicotiana benthamiana. PLoS One 8: e75124 
Nakano M, Yoshioka H, Ohnishi K, Hikichi Y, Kiba A (2015) Cell death-inducing stresses are required for defense activation in DS1-phosphatidic acid phosphatase-silenced Nicotiana benthamiana. J Plant Physiol 184: 15-19

Poueymiro M, Cunnac S, Barberis P, Deslandes L, Peeters N, Cazale-Noel AC, Boucher C, Genin S (2009) Two type III secretion system effectors from Ralstonia solanacearum GMI1000 determine host-range specificity on tobacco. Mol Plant Microbe Interact 22: 538-550

Rentel MC, Lecourieux D, Ouaked F, Usher SL, Petersen L, Okamoto H, Knight H, Peck SC, Grierson CS, Hirt H, et al. (2004) OXI1 kinase is necessary for oxidative burst-mediated signalling in Arabidopsis. Nature 427: 858-861

Starý T, Satková P, Piterková J, Mieslerová B, Luhová L, Mikulík J, Kašparovský T, Petřivalský M, Lochman J (2019) The elicitin $\beta$-cryptogein's activity in tomato is mediated by jasmonic acid and ethylene signalling pathways independently of elicitin-sterol interactions. Planta 249: 739-749

Testerink CT, Munnik T (2005) Phosphatidic acid: A multifunctional stress signaling lipid in plants. Trends Plant Sci 10: $368-375$

Torres MA (2010) ROS in biotic interactions. Physiol Plant 138:
414-429

Xue H, Chen X, Li G (2007) Involvement of phospholipid signaling in plant growth and hormone effects. Curr Opin Plant Biol 10: 483-489

Yamaguchi T, Minami E, Ueki J, Shibuya N (2005) Elicitor-induced activation of phospholipases plays an important role for the induction of defense responses in suspension-cultured rice cells. Plant Cell Physiol 46: 579-587

Yoshioka H, Numata N, Nakajima K, Katou S, Kawakita K, Rowland O, Jones JD, Doke N (2003) Nicotiana benthamiana gp91phox homologs NbrbohA and NbrbohB participate in $\mathrm{H}_{2} \mathrm{O}_{2}$ accumulation and resistance to Phytophthora infestans. Plant Cell 15: 706-718

Yu L, Nie J, Cao C, Jin Y, Yan M, Wang F, Liu J, Xiao Y, Liang Y, Zhang W (2010) Phosphatidic acid mediates salt stress response by regulation of MPK6 in Arabidopsis thaliana. New Phytol 188: 762-773

Zhang H, Fang Q, Zhang Z, Wang Y, Zheng X (2009) The role of respiratory burst oxidase homologues in elicitor-induced stomatal closure and hypersensitive response in Nicotiana benthamiana. J Exp Bot 60: 3109-3122 